\title{
Surveillance of influenza-like illness in Belgian nursing homes
}

\author{
by \\ Jans $\mathrm{B}^{1}$, Latour $\mathrm{K}^{1}$, Broex $\mathrm{E}^{1}$, Catry $\mathrm{B}^{1}$
}

\section{Keywords}

Nursing homes, surveillance, influenza, $A(H 1 N 1) 2009$, influenza-like illness, seasonal flu

\section{Introduction}

Elderly often combine several risk factors that influence the course and severity of an influenza infection. The World Health Organization strongly recommends vaccination of elderly living in care facilities. Even if vaccine effectiveness seems to decline with age (1), several studies (2-3) show that vaccination reduces the risk for pneumonia, hospitalisation and death during an influenza epidemic. Vaccination is also important among nursing home (NH) staff in close contact with this frail population. Studies have provided evidence that $\mathrm{NHs}$ with high vaccination coverage among health care workers (HCW) have a lower mortality and morbidity among residents (4-7). Belgian data on the incidence of influenza-like illness (ILI) and on vaccination among $\mathrm{NH}$ residents and $\mathrm{HCW}$ are scarce. Hence, during the $A(H 1 N 1) 2009$ pandemic, a small pilot sentinel network of $\mathrm{NHs}$ was set up in order to collect epidemiological data on ILI and vaccination coverage among $\mathrm{NH}$ residents and $\mathrm{HCW}$.

\section{Methods}

Aims of this surveillance

- to measure the incidence of ILI in Belgian NH residents during the influenza season of 2009-2010,

- to collect quantitative data on vaccination coverage for $A(H 1 N 1) 2009$ and seasonal flu as well as on absenteeism for ILI among HCW in NHs during the same observation period. 


\section{Setup of the surveillance}

The Scientific Institute of Public Health (WIV-ISP) invited all Belgian NHs $(n=1,606)$ to voluntarily participate in a national network for the surveillance of all new cases of ILI. A local surveillance contact person was designated in each participating facility.

The enrolment form completed by the local contact person provided general descriptive information on the $\mathrm{NH}$ such as the total bed capacity and the number of staff members employed. The latter group was split up in four professional categories: nursing/care staff, paramedical, logistic and administrative staff.

\section{Case definition of ILI}

ILI was defined as a condition including some or all of the following symptoms: fever, chills, myalgias, vomiting, headache, loss of appetite and/or cough. At the start of the surveillance, virological confirmation of $A(H 1 N 1) 2009$ cases was no longer routinely performed, making it impossible to distinguish between $\mathrm{A}(\mathrm{H} 1 \mathrm{~N} 1) 2009$ and seasonal flu.

\section{Collected data}

The collected resident data for each new ILI case were: year of birth, date of onset of the first symptoms, prescribed treatment (antiviral, antibiotic or other), presence of comorbidity (diabetes, chronic pulmonary disease, impaired immunity, severe/moderate cardiac illness, renal disease or hepatic disease), course of illness (recovered, hospitalised, complication, death attributable to ILI or death by other cause) and presence of an earlier ILI episode since June 2009 .

The total number of resident-days during the period was collected as denominator.

Following data concerning the $\mathrm{NH}$ staff were registered at the end of each observation period: the cumulative number of staff members by professional category that have been vaccinated against $A(H 1 N 1) 2009$ and seasonal flu and the total number of staff members by professional category absent for ILI (or complications).

\section{Surveillance period}

The surveillance period (1 November 2009 - 31 March 2010) was split into ten observation periods of 2 weeks each (from the $1^{\text {st }}$ until the $14^{\text {th }}$ and from the $15^{\text {th }}$ until the end of each month). All enrolled NHs were invited to participate throughout all 10 periods in order to obtain data covering the whole observation period. When no cases of ILI were registered during the surveillance period, the surveyor had to indicate " $0 \%$ incidence" in a specific data field. If this information was missing, participants were contacted by mail or phone in order to confirm the 'zero incidence' reporting. 


\section{Data transmission}

Participants used the available electronic datasheet or a paper form questionnaire which was optically read. At the end of each observation period, a reminder for data delivery was sent to the NHs.

\section{Calculated indicators}

The following indicators were calculated:

- The total number of new ILI cases per 1000 resident-days (incidence density) during the observation period. Missing resident-days per period were replaced by an estimated number of resident-days, based on the total $\mathrm{NH}$ bed capacity and an estimated bed occupancy rate of $98 \%$ corresponding with the average rate observed in other recent Belgian $\mathrm{NH}$ studies.

- For each professional category:

- the proportion of staff members absent for ILI (or complications);

- the proportion of staff members vaccinated against $A(H 1 N 1) 2009$ and seasonal flu.

Data were processed using STATA version 9.

\section{Ethical issues}

To assure confidentiality, the WIV-ISP provided a unique $\mathrm{NH}$ study number to each participating facility. The local contact person allotted a unique resident study number to each ILI case. Resident names were not communicated to the WIV-ISP.

\section{Results}

Participation

A total of $28 \mathrm{NHs}$ delivered surveillance data. Three facilities were excluded since resident data were missing for some surveillance periods. Finally, $25 \mathrm{NHs}$ representing 2,483 NH beds (mean: 99.3 beds/ $\mathrm{NH}$, range: $30-193$ ) and 366,605 resident-days provided surveillance data covering the 10 observation periods.

Surveillance of ILI among NH residents

\section{Total number of reported ILI cases and incidence density}

A total of 47 residents developed an ILI episode during the surveillance period. The global ILI incidence density reached $0.13 \mathrm{ILI}$ cases/1000 resident-days (Figure 1 ). The first case occurred on 2 November 2009, the last one on 16 February 2010. In 19 out of 25 participating $\mathrm{NHs}(76 \%)$, zero cases of ILI were registered. All 47 reported ILI cases occurred in 6 facilities (range: 1-30 cases/NH). Four of these facilities reported only one single ILI case occurring 
early in this ILI surveillance (between 3 November and 3 December: sporadic cases, Figure 1).

Most ILI cases (91.5\%) were clustered in $2 \mathrm{NHs}$ with a global incidence density of 1.82 cases/1000 resident-days ( $n=13$ cases) in $\mathrm{NH}-1$ (epidemic $\mathrm{NH}-1$, Figure 1 ) and 1.79/1000 resident-days ( $\mathrm{n}=30$ cases) in $\mathrm{NH}-2$ (epidemic $\mathrm{NH}-2$, Figure 1).

Figure 1. Incidence of influenza-like illness (ILI) among residents in a sentinel nursing home network November 2009-March 2010

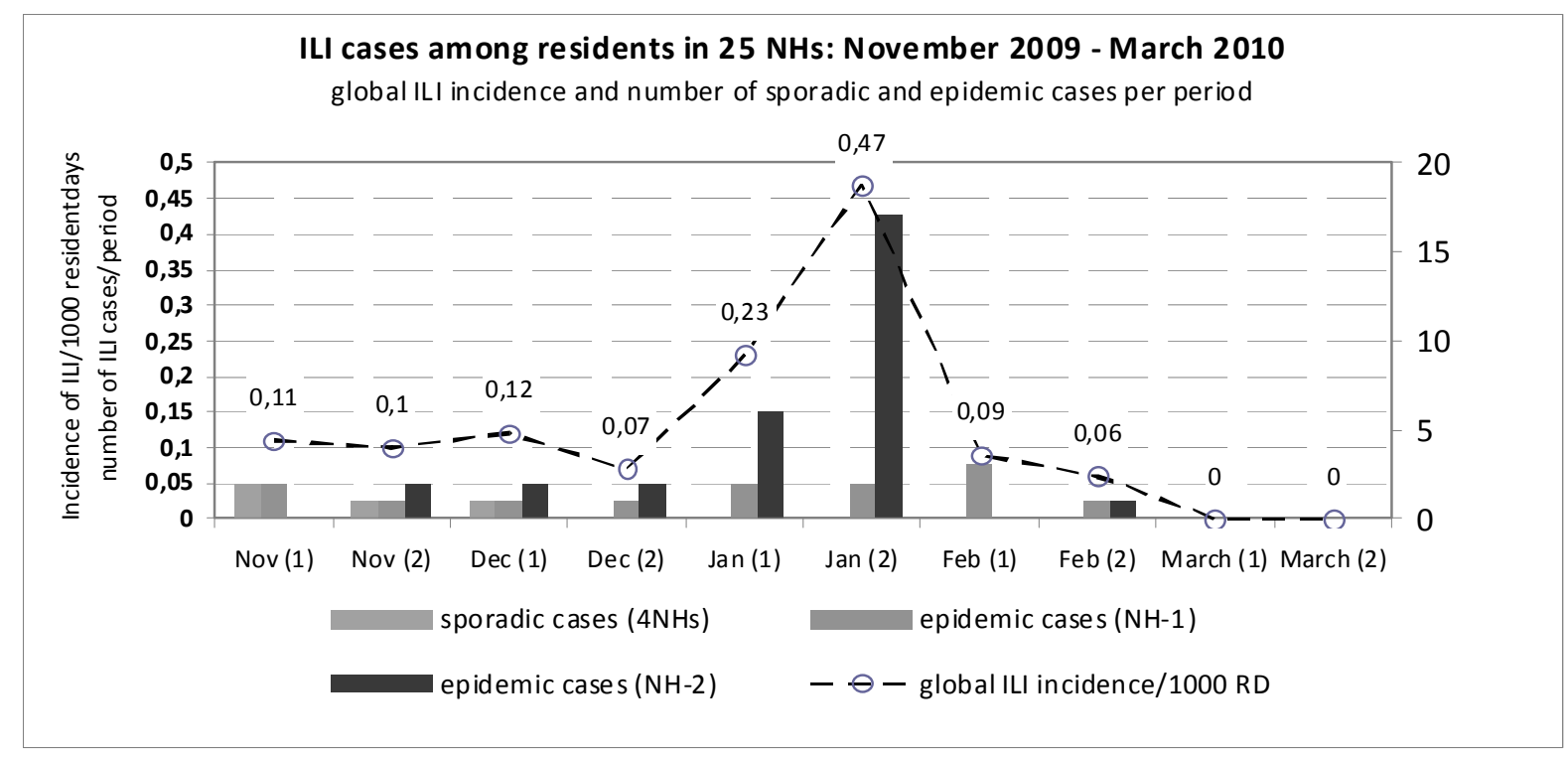

sporadic cases: $<2$ ILI cases/NH/season epidemic cases: $\geq 2$ ILI cases/NH/season

\section{Characteristics of residents with ILI}

Residents with ILI had a mean age of 85.6 years (range: $70-101)$. Comorbidity (risk factors) was present in $80.9 \%(n=38)$ of all ILI cases and was often multiple since 23 out of 38 residents at risk $(60.5 \%)$ cumulated 2 or more comorbid conditions. The main risk factors in descending order were moderate cardiac illness $(n=21)$, immunodeficiency $(n=16)$, chronic pulmonary disease $(n=13)$, moderate renal disease $(n=10)$, diabetes $(n=9)$ and chronic hepatic disease $(n=6)$.

Among the residents with ILI, 95.7\% were vaccinated against seasonal flu and $87.2 \%$ against $A(H 1 N 1) 2009$. For 4 out of 6 residents not vaccinated against $A(H 1 N 1) 2009$, ILI appeared between 2 and 6 November, just before the official $A(H 1 N 1) 2009$ vaccination campaign started in Belgian NHs (7 November 2009).

Two residents with ILI were treated with an antiviral drug, while 35 residents $(74.5 \%)$ received an antibiotic. Nine residents with ILI received another type of treatment (antipyretic, analgesic,...) and one received no treatment at all.

Five residents with ILI died (10.6\%) but according to the records only for one resident death was directly attributable to ILI. The fatal case was a 91-year-old immunodeficient resident, 
not vaccinated against $A(H 1 N 1) 2009$. All fatal cases occurred in epidemic $\mathrm{NH}-2$ which had a case fatality rate of $16.7 \%$. Complications occurred in $36.2 \%(n=17)$ of all ILI cases. Only one complicated case required hospitalisation. Of all residents with ILI, 42.5\% $(n=20)$ were admitted to the hospital. Nineteen came from epidemic $\mathrm{NH}-2$. In this $\mathrm{NH} 63.3 \%$ of all ILI cases were hospitalised. Detailed information on residents with ILI is provided in table 1.

Table 2. Characteristics of sporadic and epidemic cases of residents with influenza-like illness (ILI)

November 2009-March 2010

\begin{tabular}{|c|c|c|c|c|}
\hline Residents with ILI & $\begin{array}{l}\text { Sporadic cases } \\
(4 \mathrm{NHs})\end{array}$ & $\begin{array}{l}\text { Epidemic cases } \\
\mathrm{NH}-1\end{array}$ & $\begin{array}{l}\text { Epidemic cases } \\
\mathrm{NH}-2\end{array}$ & $\begin{array}{l}\text { Total } \\
\text { (6 NHs) }\end{array}$ \\
\hline Number of cases & 4 & 13 & 30 & 47 \\
\hline Mean age (years) & 82.8 & 89.9 & 84.1 & 85.6 \\
\hline Date of first / last ILI case & $3 \mathrm{Nov} / 3 \mathrm{Dec}$ & 2 Nov / 15 Feb & 15 Nov / 16 Feb & 2 Nov / 16 Feb \\
\hline \multicolumn{5}{|l|}{ Risk factors (comorbidity) } \\
\hline No risk factor & $1(25.0 \%)$ & $2(15.4 \%)$ & $6(20.0 \%)$ & $9(19.1 \%)$ \\
\hline Only 1 & $2(50.0 \%)$ & $5(38.5 \%)$ & $8(26.7 \%)$ & $15(31.9 \%)$ \\
\hline 2 or more & $1(25.0 \%)$ & $6(46.2 \%)$ & $16(53.3 \%)$ & $23(48.9 \%)$ \\
\hline \multicolumn{5}{|l|}{ Vaccination status } \\
\hline Seasonal flu & $4(100.0 \%)$ & $12(92.3 \%)$ & $29(96.7 \%)$ & $45(95.7 \%)$ \\
\hline $\mathrm{A}(\mathrm{H} 1 \mathrm{~N} 1) 2009$ & $2(50.0 \%)$ & $10(76.9 \%)$ & $29(96.7 \%)$ & $41(87.2 \%)$ \\
\hline \multicolumn{5}{|l|}{ Treatment for ILI } \\
\hline Antiviral only & $2(50.0 \%)$ & $0(0.0 \%)$ & $0(0.0 \%)$ & $2(4.3 \%)$ \\
\hline Antibiotic only & $1(25.0 \%)$ & $9(69.2 \%)$ & $25(83.3 \%)$ & $35(74.5 \%)$ \\
\hline Other only (antipyretic, analgesic,...) & $1(25.0 \%)$ & $3(23.1 \%)$ & $5(16.7 \%)$ & $9(19.1 \%)$ \\
\hline No treatment & $0(0.0 \%)$ & $1(7.7 \%)$ & $0(0.0 \%)$ & $1(2.1 \%)$ \\
\hline \multicolumn{5}{|l|}{ Clinical issue $^{\S}$} \\
\hline Deceased $^{*}$ & $0(0.0 \%)$ & $0(0.0 \%)$ & $5(16.7 \%)$ & $5(10.6 \%)$ \\
\hline Hospitalised & $0(0.0 \%)$ & $1(7.7 \%)$ & $19(63.3 \%)$ & $20(42.6 \%)$ \\
\hline Complication & $1(25.0 \%)$ & $11(84.6 \%)$ & $5(16.7 \%)$ & $17(36.2 \%)$ \\
\hline
\end{tabular}

$\S$ not mutually exclusive

* 1 death directly attributable to ILI 
Surveillance of ILI among NH staff

Data on absenteeism for ILI (or complication) and vaccination coverage for seasonal flu and $\mathrm{A}(\mathrm{H} 1 \mathrm{~N} 1) 2009$ among nursing home workers were available for $20 \mathrm{NHs}$.

\section{Absenteeism for ILI}

The global mean proportions of staff members absent for ILI during the 5 months of follow-up were not significantly different between professional categories and reached $4.5 \%$ among nursing staff ( $\mathrm{n}=45$ cases from $10 \mathrm{NHs}, 95 \% \mathrm{Cl}: 3.61-5.53), 5.3 \%$ in the paramedical staff ( $n=6$ cases from $1 \mathrm{NH}, 95 \% \mathrm{Cl}: 4.28-6.40), 4.9 \%$ among the logistic staff $(n=18$ cases from 6 $\mathrm{NHs}, 95 \% \mathrm{Cl}: 3.97-5.97)$ and $4.3 \%$ in administrative personnel $(n=7$ cases from $2 \mathrm{NHs}$, 95\% Cl: 3.43-5.31). The highest attack rates for ILI among nurses, paramedical staff and administrative personnel were registered in epidemic $\mathrm{NH}-2$ reaching $32 \%, 100 \%$ and $55 \%$ respectively.

Vaccination coverage for seasonal flu and A(H1N1)2009

Nineteen $\mathrm{NHs}$ provided data about the vaccination coverage for seasonal flu among $\mathrm{NH}$ staff members. Table 2 describes the vaccination coverage for both variants per professional category.

Table 2. Vaccination coverage for $A(H 1 N 1) 2009(n=20$ NHs) and seasonal flu $(n=19$ NHs)

by professional category during flu season 2009-2010

\begin{tabular}{|c|c|c|c|c|c|c|}
\hline \multirow[t]{2}{*}{$\begin{array}{l}\text { Professional } \\
\text { category }\end{array}$} & Staff members & \multicolumn{5}{|c|}{ Staff vaccinated for seasonal flu } \\
\hline & $\mathrm{n}$ & $\mathrm{n}$ & $\%$ & Range/NH & Mean \% & $95 \% \mathrm{Cl}$ \\
\hline Nursing care & 1010 & 380 & 37.6 & $0-87.5 \%$ & $43.7 \%$ & $40.85-46.69$ \\
\hline Paramedical & 119 & 50 & 42.0 & $0-100 \%$ & $48.5 \%$ & $45.44-51.76$ \\
\hline Logistic & 396 & 156 & 39.4 & $0-100 \%$ & $49.0 \%$ & $45.97-52.16$ \\
\hline \multirow[t]{2}{*}{ Administrative } & 77 & 30 & 39.0 & $0-100 \%$ & $53.2 \%$ & $49.92-56.54$ \\
\hline & & \multicolumn{5}{|c|}{ Staff vaccinated for $A(H 1 N 1) 2009$} \\
\hline Nursing care & 1029 & 436 & $42.4 \%$ & $0-93.3 \%$ & $38.8 \%$ & $36.14-41.52$ \\
\hline Paramedical & 122 & 40 & $32.8 \%$ & $0-100 \%$ & $35.8 \%$ & $33.22-38.52$ \\
\hline Logistic & 389 & 164 & $42.2 \%$ & $0-100 \%$ & $45.5 \%$ & $42.54-48.50$ \\
\hline Administrative & 74 & 34 & $45.9 \%$ & $0-100 \%$ & $52.2 \%$ & $49.08-55.46$ \\
\hline
\end{tabular}


The nursing staff, in closest contact with the frail elderly, had the lowest mean vaccination coverage for seasonal flu: $43.7 \%(95 \% \mathrm{Cl}$ : $40.85-46.69)$ while $48.5 \%(95 \% \mathrm{Cl}$ : $45.44-51.76)$ and $49 \%(95 \% \mathrm{Cl}: 45.97-52.16)$ of the paramedics and logistic staff members respectively were immunised. Vaccination coverage was the highest amongst administrative staff members (53.2\%, 95\% Cl: 49.92-56.54).

For $\mathrm{A}(\mathrm{H} 1 \mathrm{~N} 1) 2009$ the vaccination coverage was even lower since only $38.8 \%(95 \% \mathrm{Cl}$ : $36.14-41.52)$ and $35.8 \%(95 \% \mathrm{Cl}: 33.2-38.5 \%)$ of the nurses and paramedical staff respectively were protected $(n=20 \mathrm{NHs})$. The administrative and logistic staff had the highest mean vaccination coverage: $52.2 \%(95 \% \mathrm{Cl}: 49.08-55.46)$ and $45.5 \%(42.54-48.50)$ respectively.

Surprisingly, in NHs which reported residents with ILI during the flu season, the vaccination coverage for $\mathrm{A}(\mathrm{H} 1 \mathrm{~N} 1) 2009$ amongst nurses was higher $(44.8 \%, 95 \% \mathrm{Cl}: 17.13-72.55) \mathrm{com}-$ pared to NHs without ILI cases among residents (36.3\%, 95\% Cl: 20.76-51.87).

In $\mathrm{NHs}$ with ILI cases ( $\mathrm{n}=6 \mathrm{NHs})$ nurses were also more frequently $(50.3 \%, 95 \% \mathrm{Cl}$ : $31.54-$ $68.98)$ vaccinated for seasonal flu compared to nurses in NHs without cases ( $n=14 \mathrm{NHs}$ ) (40.9\%, 95\% Cl: 26.94-54.82). These differences were not statistically significant and confidence intervals were often large due to small numbers in some subcategories.

\section{Discussion}

During the flu season 2009-2010 the incidence of ILI cases among NH residents was very low and similar to trends observed in the general population. The epidemic curve of participating $\mathrm{NHs}$ showed three distinct phenomena:

- A zero ILI prevalence for $19 \mathrm{NHs,}$

- Four NHs with only one ILI case during the flu season. These sporadic cases emerged early when some $\mathrm{NH}$ residents were not yet vaccinated for $A(H 1 N 1) 2009$,

- Two facilities with an ILI epidemic (43 of 47 total ILI cases) with the highest peak at the end of January 2010. These epidemic cases emerged spread over a large period of time and extinguished mid February 2010.

Despite important vaccination rates for both influenza variants among cases, these residents developed an ILI episode anyway. Some of the early ILI cases, which occurred in vaccinated residents, could be explained by the short time span between vaccination and the onset of the ILI. Also, the 'antibody response' induced by vaccination among elderly is described to be lower (clinical vaccine efficacy: 17-53\%) than among younger adults (clinical efficacy: 70$90 \%)(8)$.

Risk factors for the acquisition of ILI were frequently present and often multiple among residents. This may explain why $75 \%$ of the cases received an antibiotic treatment, which is not advisable for viral infections in the general population. In epidemic $\mathrm{NH}-2$, the proportion of residents receiving antibiotics was even higher (83\%). Another possible explanation for the 
important proportion of ILI cases receiving antibiotics could be the relatively high number of cases with a complications, especially in epidemic $\mathrm{NH}-1$ reaching $84.6 \%$.

In epidemic $\mathrm{NH}-2$ the hospitalisation rate of cases with ILI was surprisingly high $(63.3 \%)$ despite a relatively low number of complications (16.7\%). In this same $\mathrm{NH}$ the mortality among ILI cases was important (16.7\%), but according to the records only for one resident death was directly attributable to ILI. This very aged, immunocompromised resident was not vaccinated against $\mathrm{A}(\mathrm{H} 1 \mathrm{~N} 1) 2009$. A different vaccination strategy for $\mathrm{NH}$ residents in terminal or palliative care could be an explanation for this, but further investigation is needed.

In participating $\mathrm{NHs}$, the vaccination coverage rate for seasonal flu and for $\mathrm{A}(\mathrm{H} 1 \mathrm{~N} 1) 2009$ among HCWs directly involved in resident care (nurses) was low: $38.8 \%$ and $43.7 \%$ on average. In scientific literature vaccination rates of less than $50 \%$ are mentioned (9).

The vaccination coverage for both variants was better among administrative staff members than among nurses.

During this flu season the mean proportion of HCW on sick leave for ILI was not significantly different between the professional categories, but varied strongly by $\mathrm{NH}$. In epidemic $\mathrm{NH}-2$, the total paramedical staff, half of the administrative personnel and one third of the nursing care staff was absent for ILI.

This surveillance provided interesting data, but the relatively small study sample (25 NHs) and the low incidence of ILI did not allow us to investigate in more detail the possible relationship between vaccination coverage among nurses and the incidence of ILI and associated complications and mortality among residents.

One of the difficulties in this surveillance was to convince NHs to send data while cases were scarce or absent. Another weakness was the absence of vaccination coverage data for both influenza variants in the total $\mathrm{NH}$-population.

'Resident-days' as a denominator for calculation of the ILI incidence was often missing and replaced by an estimated number of resident-days assuming that the bed occupancy rate was comparable between institutions. Other data were also difficult to obtain: e.g. if vaccination of an HCW was carried out by the personal general practitioner, the institution did not record this. Finally, several participants were not able to provide data on absenteeism of $\mathrm{HCW}$ for ILI, since the reasons for sick leave of HCWs were confidential.

Strengths of this surveillance were the low associated workload, the 'easy-to-use' methodology.

This continuous ILI surveillance in NHs provides important data on the severity degree of ILI in the population with the highest risk in terms of morbidity and mortality, namely elderly living in collectivity in which vaccination is less effective due to immunosenescence. The results of this surveillance could lead to a more rational and specific management of ILI in these facilities. 


\section{Acknowledgements}

The authors would like to express their gratitude to all nursing homes that kindly collected data for this surveillance.

\section{References}

1. Thijs C, Beyer WE, Govaert PM, Sprenger MJ, Dinant GJ, Knottnerus A. Mortality benefits of influenza vaccination in elderly people. Lancet Infect Dis 2008; 8(8):460-1

2. Patriarca PA, Weber JA, Parker RA, Hall WN, Kendal AP, Bregman DJ et al. Efficacy of influenza vaccine in nursing homes. Reduction in illness and complications during an influenza $A(H 3 N 2)$ epidemic. JAMA 1985; 253(8):1136-9

3. Deguchi $\mathrm{Y}$, Takasugi $\mathrm{Y}$, Tatara K. Efficacy of influenza vaccine in the elderly in welfare nursing homes: reduction in risks of mortality and morbidity during an influenza A (H3N2) epidemic. J Med Microbiol 2000; 49(6):553-6

4. Carman WF, Elder AG, Wallace LA, McAulay K, Walker A, Murray GD et al. Effects of influenza vaccination of health-care workers on mortality of elderly people in long-term care: a randomised controlled trial. Lancet 2000; 355(9198):93-7

5. Drinka PJ, Gravenstein S, Krause P, Schilling M, Miller BA, Shult P. Outbreaks of influenza A and B in a highly immunized nursing home population. J Fam Pract 1997; 45(6):509-14

6. Potter J, Stott DJ, Roberts MA, Elder AG, O'Donnell B, Knight PV et al. Influenza vaccination of health care workers in long-term-care hospitals reduces the mortality of elderly patients. J Infect Dis 1997; 175(1):1-6

7. Hayward AC, Harling R, Wetten S, Johnson AM, Munro S, Smedley J et al. Effectiveness of an influenza vaccine programme for care home staff to prevent death, morbidity, and health service use among residents: cluster randomised controlled trial. BMJ 2006; 333(7581):1241

8. Goodwin K, Viboud C, Simonsen L. Antibody response to influenza vaccination in the elderly: a quantitative review. Vaccine 2006; 24(8):1159-69

9. Sartor C, Tissot-Dupont H, Zandotti C, Martin F, Roques $P$, Drancourt M. Use of a mobile cart influenza program for vaccination of hospital employees. Infect Control Hosp Epidemiol 2004; 25(11):918-22 